\title{
Group-based cognitive-behavioural anger management for people with mild to moderate intellectual disabilities: cluster randomised controlled trial ${ }^{\dagger}$
}

Paul Willner, John Rose, Andrew Jahoda, Biza Stenfert Kroese, David Felce, David Cohen, Pamela MacMahon, Aimee Stimpson, Nicola Rose, David Gillespie, Jennifer Shead, Claire Lammie, Christopher Woodgate, Julia Townson, Jacqueline Nuttall and Kerenza Hood

\section{Background}

Many people with intellectual disabilities find it hard to control their anger and this often leads to aggression which can have serious consequences, such as exclusion from mainstream services and the need for potentially more expensive emergency placements.

\begin{abstract}
Aims
To evaluate the effectiveness of a cognitive-behavioural therapy (CBT) intervention for anger management in people with intellectual disabilities.
\end{abstract}

\begin{abstract}
Method
A cluster-randomised trial of group-based 12-week CBT, which took place in day services for people with intellectual disabilities and was delivered by care staff using a treatment manual. Participants were 179 service users identified as having problems with anger control randomly assigned to either anger management or treatment as usual.

Assessments were conducted before the intervention, and at 16 weeks and 10 months after randomisation (trial registration: ISRCTN37509773).
\end{abstract}

\section{Results}

The intervention had only a small, and non-significant, effect on participants' reports of anger on the Provocation Index, the primary outcome measure (mean difference $2.8,95 \% \mathrm{Cl}$ -1.7 to 7.4 at 10 months). However, keyworker Provocation Index ratings were significantly lower in both follow-up assessments, as were service-user ratings on another selfreport anger measure based on personally salient triggers. Both service users and their keyworkers reported greater usage of anger coping skills at both follow-up assessments and keyworkers and home carers reported lower levels of challenging behaviour.

\section{Conclusions}

The intervention was effective in improving anger control by people with intellectual disabilities. It provides evidence of the effectiveness of a CBT intervention for this client group and demonstrates that the staff who work with them can be trained and supervised to deliver such an intervention with reasonable fidelity.

\section{Declaration of interest}

None.

mild-to-moderate intellectual disabilities that was provided in the participants' day services. There is some evidence that service users accompanied to the group by care staff achieve better outcomes, ${ }^{15}$ and this is now considered to be best practice in learning disability services. There is also some evidence that not only qualified clinicians, but also care staff can effectively deliver manualised interventions for depression ${ }^{13}$ and anger ${ }^{16}$ to people with intellectual disabilities. The present intervention was therefore delivered by care staff ('lay therapists') working in the services that the participants attended, ${ }^{13,16,17}$ to exploit the potential of the ongoing relationships that care staff would have with service users. A group format was chosen for several reasons: it offers potential economies of scale, some relevant activities such as role-play are easier to implement with more people present, and group work - albeit not of a psychotherapeutic nature - would be familiar to all staff and users of learning disability services.

\section{Method}

\section{Design}

This was a multicentre phase three cluster randomised controlled trial (RCT) of a manualised anger management group intervention $v$. a 'treatment as usual' waiting-list control condition, with randomisation of the group rather than the individual (trial registration: ISRCTN37509773). A power calculation indicated a 
target of 72 participants in each arm of the trial in order to detect a standardised effect size of 0.57 with $80 \%$ power at a $5 \%$ significance level, inflating for clustering with an intracluster correlation coefficient (ICC) of 0.11 . We aimed to recruit a group of 4-9 service users (an average of 6) in each of 30 participating day services, allowing for $20 \%$ loss to follow-up. Full details of the methodology, including literature references, are in the study protocol. ${ }^{18}$ Ethical approval was granted by the South-East Wales Research Ethics Committee (09/WSE03/41).

\section{Participants}

We recruited 30 services that provide day activities for people with intellectual disabilities (17 local authority and 13 non-local authority providers), in parts of Scotland, England and Wales. Within each service, four to eight service users were recruited. Participants were included on the basis that they were identified by service staff as having problems in managing anger, wished to learn to improve their anger management skills and were able to provide informed consent and complete the assessments. Potential participants were excluded if they were attending the service for a reason other than a diagnosed intellectual disability or were currently receiving or requiring an urgent referral for individual treatment of anger or aggression or were experiencing circumstances indicating that a Protection of Vulnerable Adults procedure should be initiated. As is usual for people with intellectual disabilities attending day services, participants' intellectual disabilities were of varying aetiology, which typically was not recorded.

For each service user, a keyworker (the staff member with primary responsibility for the individual), and, where applicable, a home carer were also recruited. In each participating centre, between two and four staff were recruited to act as lay therapists. Staff were nominated by their managers and selected, without reference to formal qualifications, on the basis of their motivation to take on the lay therapist role and their openness to use a cognitive-behavioural approach.

\section{Randomisation, timing of data collection and masking}

Baseline data were collected from all participating service users and their keyworkers before randomisation took place. Randomisation of centres was performed using the method of minimisation, with a random component, set at $80 \%$. Centres were balanced on: (a) their service users' average baseline self-reported Provocation Index score (see later), (b) the number of service users recruited and (c) the average number of hours a week spent by the service user with at least one lay therapist outside of sessions.

Quantitative assessments were administered before randomisation, in a 2-week window beginning 16-weeks after randomisation, and again in a 4-week window 10 months after randomisation. The researchers undertaking the outcome assessments had no involvement in training or supervision of the therapists and were in principle masked to the group allocation of the service. In practice, the assessors were sometimes made aware of group allocation by service users, although this was never confirmed to them by other members of the research team.

\section{Intervention}

Participants in services randomised to the intervention arm of the trial received a manualised CBT intervention, consisting of 12 weekly psychoeducational group sessions supplemented by 'homework'. The intervention was essentially as previously described, ${ }^{17}$ with some minor modifications to the order of some of the elements. It was constructed from a CBT orientation but did not include the full range of CBT techniques, some of which (e.g. Socratic questioning) would be difficult to ask of minimally trained therapists. After randomisation and before the start of the intervention, a clinical psychologist provided the lay therapists with three training sessions, delivered over the course of a single day, covering the principles of anger management and use of the therapy manual. The clinical psychologist subsequently supervised the lay therapists fortnightly.

The fidelity of delivery of the intervention was monitored by pairs of observers who scored two sessions for each group according to a fidelity-monitoring instrument developed within the project.

\section{Baseline and outcome measures}

Participants' intellectual and receptive language abilities were assessed at baseline using the Wechsler Abbreviated Scale of Intelligence (WASI) ${ }^{19}$ and the British Picture Vocabulary Scale $(\text { BPVS-III })^{20}$ respectively. Adaptive behaviour was assessed using the short form of the Adaptive Behavior Scale (ABS), ${ }^{21}$ which was completed by the service-user's keyworker or home carer.

The primary outcome measure was the Provocation $\operatorname{Index}^{3}$ as completed by the service user at 10-month follow-up, a direct measure of felt response to hypothetical, potential angerprovoking situations that has frequently been used with this service-user group. The Provocation Index was also completed by a keyworker and a home carer. For this and other measures, in the event that a service-user's keyworker was involved in the trial as a lay therapist, then wherever possible, the measure was completed by another staff member in the service. The Provocation Index has been reported to show excellent internal consistency (Cronbach's alpha 0.95). ${ }^{3}$ For the present sample, computed alpha values for service users, keyworkers and home carers were 0.92 , 0.90 and 0.90 respectively.

The Profile of Anger Coping Skills (PACS ${ }^{17}$ was completed by service user, keyworker and home carer, to assess the development of functional coping skills. The PACS is based on three scenarios identified as reliable triggers to anger for each individual service user. The service-user version of the PACS includes a self-rating of the anger evoked by each situation, which we refer to as the PACS Imaginal Provocation Test (PACS-IPT). It differs from the Provocation Index in relating to actual situations known to provoke anger, rather than to hypothetical situations.

Mental health was assessed by self-report using the Glasgow Depression and Anxiety Scales (GDS/GAS), ${ }^{22,23}$ which are established measures of depression and anxiety among people with intellectual disability and an adaptation of the Rosenberg Self-Esteem Scale (RSES) ${ }^{24}$ for people with intellectual disability. Self-reported quality of life was assessed using the Comprehensive Quality of Life Scale - Intellectual Disability (ComQoL-ID). ${ }^{25}$

Challenging behaviour was assessed by keyworker and homecarer report using the Hyperactivity and Irritability domain items of the Aberrant Behavior Checklist (ABC-H/I) ${ }^{26}$ which address a wide range of aggressive, self-injurious, destructive and overactive challenging behaviours, and the Modified Overt Aggression Scale (MOAS), ${ }^{27}$ which is more specifically focused on aggressive challenging behaviour.

A cost and consequences analysis established the costs of delivering the intervention and its impact on health and social care resource use. All resource inputs in delivering the intervention (for example psychology, administrative and lay therapist time, related travel and consumables) were recorded prospectively using logs developed for the study, which also recorded the details of each group session and whether group participants would ordinarily have attended the day service at that time (i.e. received 
treatment as usual) or not. All other health and social care service inputs consumed by study participants in the 12 weeks prior to baseline and prior to the 10-month follow-up were ascertained using an adapted version of the Client Service Receipt Inventory. ${ }^{28}$

\section{Data analysis}

The primary analysis was based on the intention-to-treat principle and compared the mean self-reported Provocation Index between the two groups using a two-level analysis of covariance (ANCOVA) model, to allow for clustering within centres, with participants at level 1 and centres at level 2, and with baseline levels of the Provocation Index as a covariate.

Secondary outcomes were analysed similarly, with some outcomes transformed to improve model fit. Transformations did not improve the fit for the MOAS home-carer 10-month ratings model so ranked $\mathrm{ANCOVA}^{29}$ was used to analyse this outcome. Effect sizes (Cohen's $d$ ) were calculated as standardised mean differences, derived from the $F$-statistic of the two-level ANCOVA models. ${ }^{30}$

A complier-adjusted causal effect (CACE) was estimated using an instrumental variable regression analysis to assess the impact of non-compliance with the intervention on each outcome measure. ${ }^{31}$ For the purposes of these analyses, someone who had attended at least 8 of the 12 sessions was considered to be adherent to treatment.

A post hoc analysis of PACS-IPT scores was conducted using a ranked ANCOVA, based on the median anger rating of each participant across the three PACS scenarios. For the health economic analysis, resources were valued using unit costs for the financial year 2010-2011 from sources obtainable from the authors. The costs of resources involved in delivering the intervention were summed and divided by the sum of the product of the number of participants in each group session and its length (in hours) to produce the cost per person-hour. The incremental cost of delivering the intervention was calculated by deducting an estimate of the direct care cost per hour of local authority day care (treatment as usual), taking account of the extent to which the intervention replaced treatment as usual.

A total weekly cost of health and social care service use per participant was calculated at baseline and 10-month follow-up. Differences in the costs between the intervention and control groups at 10-month follow-up, taking account of baseline levels, were explored using ANCOVA. An initial two-level (service users nested within services) ANCOVA analysis detected no clustering at the service level. Therefore a single-level ANCOVA model was fitted. The 95\% confidence intervals were calculated using nonparametric bootstrapping with a total of 5000 replications, using the cluster command to account for any correlation at service level, and with the random seed number set at five.

\section{Results}

\section{Recruitment and participant flow}

Centres were recruited between December 2009 and September 2010, and service users between February and September 2010. The main intervention started in April 2010 and ended in February 2011, with 10-month follow-up assessments ending in September 2011. A consort diagram is shown in Fig. 1. A total of 30 services (179 service users) were randomised. There was a $9 \%$ withdrawal rate at the 16-week follow-up, with a further $10 \%$ withdrawing over the next 6 months, giving an overall withdrawal rate at the 10-month follow-up of $19 \%$ (1 service and 18 service users in each of the intervention and control conditions).
Demographic details for all groups of participants are shown in Table 1. Most service users lived in the family home (41\%) or in staffed group homes or supported living (38\%): 7\% lived in hospital accommodation and 9\% lived independently. Service users had high levels of psychopathology, with 34\% and 73\% scoring above clinical cut-off criteria for depression and anxiety respectively, and $26 \%$ scoring above a criterion that has been used to identify severe challenging behaviour. ${ }^{32}$ There are unfortunately no normative data against which to compare the levels of anger in this sample. A total of $21 \%$ of participants $(17 \%$ of the intervention group and $25 \%$ of the control group) were receiving antidepressant and/or antipsychotic medication (7\% and 15\% respectively).

The control and intervention arms of the trial were well matched on the variables on which randomisation was based: mean Provocation Index, 44 (s.d. $=15)$ and 43 (s.d.=19); mean number of service users per group, $6.0($ s.d.=5.7) and 6.0 $($ s.d. = 5.7); median hours per week spent with at least one trainer, $6.7(\mathrm{IQR}=3.0-8.6)$ and $7.8(\mathrm{IQR}=4.7-17.0)$. The two arms of the trial were also well matched on all demographic (Table 1) and clinical (Table 2) variables.

A total of 37 lay therapists were trained to deliver the intervention. They had worked with people with intellectual disabilities for a median of 9 years. Only 16\% $(n=6)$ had a university or equivalent-level education; $32 \%(n=12)$ had low (NVQ level 1 or similar) or no educational qualifications and $52 \%(n=19)$ had intermediate qualifications. None had received any formal training in CBT.

\section{Fidelity of the intervention}

Fidelity was assessed as relatively good overall (mean rating $686 \%$ ), but varied widely across centres (range $40-86 \%$, with the exception of one group rated at 19\%). Elements of the intervention relating to group process and behavioural coping strategies were delivered better than elements relating to participants' emotions and cognitions. Global ratings of fidelity to the manual, to group process and to the principles of CBT were $73 \%, 74 \%$ and $57 \%$ respectively. These data will be reported in more detail elsewhere.

\section{Outcomes}

\section{Anger}

On the primary outcome, self-reported Provocation Index scores at 10-month follow-up, the intervention effect was in the predicted direction, but not statistically significant (Table 3 ). The effect was larger at the 16-week follow-up (Table 3) and in the CACE analysis $(P=0.063)$, although neither achieved significance.

Service users' mean baseline ratings on the Provocation Index were just under $60 \%$ of maximum. Baseline PACS-IPT ratings were higher ( $85 \%$ maximum), as expected given that these scenarios represented individualised triggers for anger. The PACS-IPT ratings decreased significantly in the intervention group at the 16-week follow-up $(P<0.01)$, and the difference between intervention and control groups was maintained at the 10-month follow-up $(\mathrm{P}<0.05)$ (Table 4).

Keyworkers, but not home carers, reported lower Provocation Index scores in the intervention group. The effect was nonsignificant at 16 -week follow-up $(P=0.060)$ but significant at 10-month follow-up ( $P=0.023$; Table 3$)$, and was significant at both time points in the CACE analysis (16 weeks: $P=0.041$; 10 months: $P=0.009)$. The effects of the intervention were not 


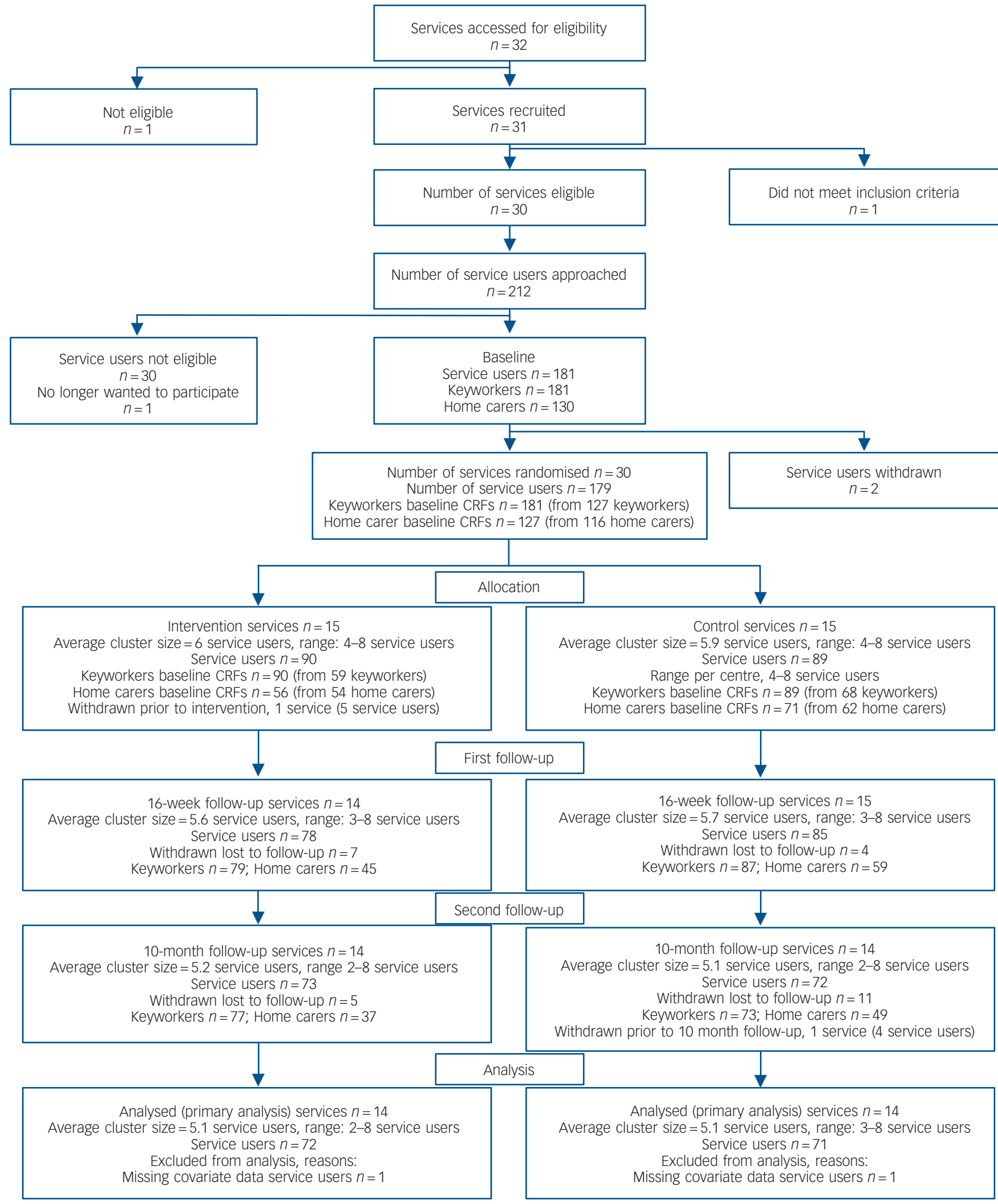

Fig. 1 Consort diagram.

CRFs, case report forms.

diminished by excluding reports from keyworkers who were also lay therapists $(n=14)$ (Table 3$)$.

\section{Anger coping}

Service users and keyworkers, but not home carers, reported significantly higher anger coping in the intervention group at both follow-up assessments (Table 5). Service user and keyworker 16-week PACS ratings were significantly correlated in the intervention group $($ rho $=0.272, P=0.021)$, but not in the control group (rho $=0.119, P=0.289$ ).

\section{Challenging behaviour}

Keyworkers reported significantly better outcomes at 16-week follow-up on two of the three measures of challenging behaviour, 
Table 1 Participants' demographic details

\begin{tabular}{|c|c|c|c|c|c|}
\hline & \multicolumn{2}{|c|}{ Service users } & \multirow[b]{2}{*}{$\begin{array}{c}\text { Keyworkers }^{\text {a }} \\
\quad(n=124)\end{array}$} & \multirow[b]{2}{*}{$\begin{array}{l}\text { Lay therapists } \\
\qquad(n=37)\end{array}$} & \multirow[b]{2}{*}{$\begin{array}{l}\text { Home carers } \\
\qquad(n=127)\end{array}$} \\
\hline & $\begin{array}{l}\text { Control } \\
(n=90)\end{array}$ & $\begin{array}{l}\text { Intervention } \\
\quad(n=91)\end{array}$ & & & \\
\hline Age, years: median (IQR) & $38.5(28.0-46.0)$ & $37.0(27.5-48.5)$ & $46(40-52)$ & $46(36-51)$ & $50(34-58)$ \\
\hline Gender, \% male & 70.0 & 71.4 & 40 & 49 & 35 \\
\hline IQ, median (IQR) & $55.0(53.0-59.0)$ & $59.0(55.0-64.0)$ & & & \\
\hline Adaptive Behavior Scale score, mean (s.d.) & $84.9(15.8)$ & $84.5(15.3)$ & & & \\
\hline
\end{tabular}

\begin{tabular}{|c|c|c|c|c|c|c|}
\hline \multirow[b]{2}{*}{ Measure } & \multicolumn{2}{|c|}{ Service user } & \multicolumn{2}{|c|}{ Keyworker } & \multicolumn{2}{|c|}{ Home carer } \\
\hline & Control & Intervention & Control & Intervention & Control & Intervention \\
\hline Provocation Index, mean (s.d.) & $44(15)$ & $43(19)$ & $33(14)$ & $32(12)$ & $30(13)$ & $31(14)$ \\
\hline Profile of Anger Coping Skills, median (IQR) & $25(16-41)$ & $25(15-38)$ & $11(7-21)$ & $13(4-21)$ & $14(7-19)$ & $7(1-17)$ \\
\hline Glasgow Depression Scale, mean (s.d.) & $9\left(5,13^{\mathrm{a}}\right)$ & $10\left(6,16^{a}\right)$ & & & & \\
\hline Glasgow Anxiety Scale, mean (s.d.) & $18(9)$ & $19(10)$ & & & & \\
\hline Rosenberg Self-Esteem Scale, median (IQR) & $26(22-29)$ & $25(22-28)$ & & & & \\
\hline Comprehensive Quality of Life, median (IQR) & $105(76-125)$ & $100(77-129)$ & & & & \\
\hline $\begin{array}{l}\text { Aberrant Behavior Checklist - Hyperactivity, } \\
\text { median (IQR) }\end{array}$ & & & $8(4-14)$ & $9(5-17)$ & $10(4-14)$ & $8(4-18)$ \\
\hline $\begin{array}{l}\text { Aberrant Behavior Checklist - Irritability, } \\
\text { median (IQR) }\end{array}$ & & & $9(4-15)$ & $9(5-15)$ & $10(6-18)$ & $12(4-21)$ \\
\hline Modified Overt Aggression Scale, median (IQR) & & & $9(3-20)$ & $8(4-22)$ & $10(1-23)$ & $11(3-23)$ \\
\hline
\end{tabular}

\section{Table 3 Self- and carer-rated anger in hypothetical situations, assessed using the Provocation Index}

\begin{tabular}{|c|c|c|c|c|c|c|c|c|c|}
\hline \multirow[b]{2}{*}{ Rater and follow-up time } & \multicolumn{3}{|c|}{$n$} & \multicolumn{2}{|c|}{ Mean (s.d.) } & \multirow{2}{*}{$\begin{array}{l}\text { Standardised } \\
\text { effect size }\end{array}$} & \multirow{2}{*}{$\begin{array}{c}\text { Adjusted mean } \\
\text { difference }^{\mathrm{b}, \mathrm{c}}(95 \% \mathrm{Cl})\end{array}$} & \multirow[b]{2}{*}{$\mathrm{ICC}^{\mathrm{C}}$} & \multirow[b]{2}{*}{$P$} \\
\hline & Total & Control & Intervention & Control & Intervention & & & & \\
\hline \multicolumn{10}{|l|}{ Service users } \\
\hline 16 weeks & 162 & 85 & 77 & $47.8(14.81)$ & 41.5 (29.15) & 0.20 & $4.4(-0.6$ to 9.4$)$ & 0.124 & 0.083 \\
\hline 10 months & 143 & 72 & 71 & $45.1(17.46)$ & $41.4(23.78)$ & 0.16 & $2.8(-1.7$ to 7.4$)$ & 0.005 & 0.210 \\
\hline \multicolumn{10}{|l|}{ Keyworkers } \\
\hline 16 weeks & 161 & 82 & 79 & $37.7(19.7)$ & $30.8(20.3)$ & 0.27 & $6.1(-0.3$ to 12.5$)$ & 0.276 & 0.060 \\
\hline 10 months & 150 & 73 & 77 & $35.7(21.6)$ & $28.6(17.3)$ & 0.34 & $6.3(0.9$ to 11.6$)$ & 0.103 & 0.023 \\
\hline \multicolumn{10}{|l|}{$\begin{array}{l}\text { Keyworkers excluding lay } \\
\text { therapist reports }\end{array}$} \\
\hline 16 weeks & 147 & 82 & 65 & 37.7 (19.7) & $29.1(19.7)$ & 0.33 & 7.3 (0.7 to 13.9) & 0.284 & 0.032 \\
\hline 10 months & 136 & 73 & 63 & $35.7(21.6)$ & $27.8(16.7)$ & 0.37 & $6.9(1.1$ to 12.7$)$ & 0.133 & 0.021 \\
\hline \multicolumn{10}{|l|}{ Home carers } \\
\hline 16 weeks & 104 & 59 & 45 & $34.0(16.5)$ & $31.4(14.6)$ & 0.13 & $1.9(-2.5$ to 6.2$)$ & 0.005 & 0.372 \\
\hline 10 months & 84 & 41 & 43 & $27.8(17.6)$ & $29.3(15.9)$ & 0.02 & $0.3(-6.6$ to 7.1$)$ & 0.000 & 0.940 \\
\hline
\end{tabular}

\begin{tabular}{|c|c|c|c|c|c|c|}
\hline \multirow[b]{2}{*}{ Median rating ${ }^{a}$} & \multicolumn{2}{|c|}{ Baseline, \% (n) } & \multicolumn{2}{|c|}{ 16-week follow-up, \% (n) } & \multicolumn{2}{|c|}{ 10-month follow-up, \% (n) } \\
\hline & Control & Intervention & Control & Intervention & Control & Intervention \\
\hline $0-0.5$ & $3.6(3)$ & $3.4(3)$ & $3.7(3)$ & $18.4(14)$ & $12.1(8)$ & $29.6(21)$ \\
\hline $1-1.5$ & $31.0(26)$ & $25.9(23)$ & $28.1(23)$ & $26.3(20)$ & $42.4(28)$ & $29.6(21)$ \\
\hline 2 & $65.5(55)$ & $70.8(63)$ & $68.3(56)$ & $55.3(42)$ & $45.5(30)$ & 40.8 (29) \\
\hline
\end{tabular}




\begin{tabular}{|c|c|c|c|c|c|c|c|c|c|}
\hline \multirow[b]{2}{*}{ Rater and follow-up time } & \multicolumn{3}{|c|}{$n$} & \multicolumn{2}{|c|}{ Mean (s.d.) } & \multirow{2}{*}{$\begin{array}{l}\text { Standardised } \\
\text { effect size }\end{array}$} & \multirow{2}{*}{$\begin{array}{c}\text { Adjusted mean } \\
\text { difference }^{b, c}(95 \% \mathrm{Cl})\end{array}$} & \multirow[b]{2}{*}{$I C C^{C}$} & \multirow[b]{2}{*}{$P$} \\
\hline & Total & Control & Intervention & Control & Intervention & & & & \\
\hline \multicolumn{10}{|l|}{ Service users } \\
\hline 16 weeks & 156 & 82 & 74 & $29.2(24.0)$ & $37.9(25.0)$ & 0.51 & $-11.3(-17.7$ to -4.9$)$ & 0.129 & 0.001 \\
\hline 10 months & 138 & 67 & 71 & $26.4(23.2)$ & $34.1(27.2)$ & 0.43 & $-9.7(-16.8$ to -2.6$)$ & 0.139 & 0.010 \\
\hline \multicolumn{10}{|l|}{ Keyworkers } \\
\hline 16 weeks $^{\mathrm{d}}$ & 157 & 81 & 76 & $17.1(12.2)$ & $24.1(18.4)$ & 0.53 & $-1.0(-1.6$ to -0.4 & 0.100 & 0.002 \\
\hline 10 months $^{\mathrm{d}}$ & 140 & 70 & 70 & $16.5(13.4)$ & $23.9(19.2)$ & 0.49 & $-0.9(-1.6$ to -0.3$)$ & 0.113 & 0.006 \\
\hline \multicolumn{10}{|l|}{$\begin{array}{l}\text { Keyworkers excluding lay } \\
\text { therapist reports }\end{array}$} \\
\hline 16 weeks $^{d}$ & 146 & 81 & 65 & $17.1(12.2)$ & $23.9(16.8)$ & 0.51 & $-0.9(-1.5$ to -0.3$)$ & 0.080 & 0.003 \\
\hline 10 months $^{d}$ & 128 & 70 & 58 & $16.5(13.4)$ & $22.6(15.4)$ & 0.42 & $-0.8(-1.4$ to -0.1$)$ & 0.085 & 0.021 \\
\hline \multicolumn{10}{|l|}{ Home carers } \\
\hline 16 weeks $^{d}$ & 103 & 58 & 45 & $16.1(17.7)$ & $20.4(19.2)$ & 0.18 & $-0.5(-1.5$ to 0.6$)$ & 0.256 & 0.356 \\
\hline 10 months $^{d}$ & 85 & 42 & 43 & $19.0(21.5)$ & $19.0(20.9)$ & 0.08 & $-1.5(-11.5$ to 8.5$)$ & 0.166 & 0.749 \\
\hline $\begin{array}{l}\text { a. Standard deviation inflated fo } \\
\text { b. Difference calculated as: Con } \\
\text { c. Intracluster correlation coeffi } \\
\text { d. Adjusted mean difference an }\end{array}$ & $\begin{array}{l}\text { tre-le } \\
\text { Intery } \\
\text { (ICC): }\end{array}$ & ering. & gyare & rmation & line scor & 19: & ance (ANCOVA) & & \\
\hline
\end{tabular}

\begin{tabular}{|c|c|c|c|c|c|c|c|c|c|}
\hline \multirow[b]{2}{*}{ Outcome } & \multicolumn{3}{|c|}{$n$} & \multicolumn{2}{|c|}{ Mean (s.d.) $)^{a}$} & \multirow{2}{*}{$\begin{array}{l}\text { Standardised } \\
\text { effect size }\end{array}$} & \multirow{2}{*}{$\begin{array}{l}\text { Adjusted mean } \\
\text { difference }^{\text {b,c }}(95 \% \mathrm{Cl})\end{array}$} & \multirow[b]{2}{*}{$\mathrm{ICC}^{\mathrm{C}}$} & \multirow[b]{2}{*}{$P$} \\
\hline & Total & Contro & rvention & Control & Intervention & & & & \\
\hline \multicolumn{10}{|l|}{ Keyworkers } \\
\hline \multicolumn{10}{|l|}{ Outcomes at 16 weeks } \\
\hline Aberrant Behavior Checklist - Hyperactivity ${ }^{d}$ & 159 & 81 & 78 & $12.7(10.2)$ & $7.9(7.7)$ & 0.45 & 0.7 (0.3 to 1.1) & 0.058 & 0.001 \\
\hline Aberrant Behavior Checklist - Irritability ${ }^{d}$ & 158 & 81 & 77 & $11.0(9.5)$ & $7.5(7.8)$ & 0.37 & $0.7(0.2$ to 1.1$)$ & 0.184 & 0.005 \\
\hline Modified Overt Aggression Scale ${ }^{d}$ & 158 & 82 & 76 & $8.3(12.2)$ & $10.0(14.8)$ & 0.01 & $-0.1(-0.9$ to 0.8$)$ & 0.263 & 0.914 \\
\hline \multicolumn{10}{|l|}{ Outcomes at 10 months } \\
\hline Aberrant Behavior Checklist - Hyperactivity ${ }^{d}$ & 150 & 73 & 77 & $9.4(9.0)$ & $8.2(8.4)$ & 0.14 & $0.3(-0.2$ to 0.7$)$ & 0.116 & 0.263 \\
\hline Aberrant Behavior Checklist - Irritability ${ }^{d}$ & 150 & 73 & 77 & $7.6(6.8)$ & $8.4(9.8)$ & 0.08 & $0.1(-0.3$ to 0.6$)$ & 0.095 & 0.561 \\
\hline Modified Overt Aggression Scale ${ }^{d}$ & 140 & 66 & 74 & $5.2(12.1)$ & $5.6(12.2)$ & 0.03 & $0.1(-0.7$ to 0.8$)$ & 0.174 & 0.818 \\
\hline \multicolumn{10}{|l|}{ Home carers } \\
\hline \multicolumn{10}{|l|}{ Outcomes at 16 weeks } \\
\hline Aberrant Behavior Checklist - Hyperactivity ${ }^{d}$ & 104 & 59 & 45 & $12.1(12.0)$ & $9.3(9.7)$ & 0.32 & $0.5(0.0$ to 1.1$)$ & 0.060 & 0.046 \\
\hline Aberrant Behavior Checklist - Irritability ${ }^{d}$ & 104 & 59 & 45 & $12.4(9.6)$ & $9.3(10.9)$ & 0.22 & $0.4(-0.2$ to 1.1$)$ & 0.154 & 0.187 \\
\hline Modified Overt Aggression Scale ${ }^{d}$ & 103 & 58 & 45 & $12.7(14.7)$ & 8.7 (18.3) & 0.11 & $0.4(-0.8$ to 1.4$)$ & 0.275 & 0.520 \\
\hline \multicolumn{10}{|l|}{ Outcomes at 10 months } \\
\hline Aberrant Behavior Checklist - Hyperactivity ${ }^{d}$ & 84 & 41 & 43 & $9.1(13.8)$ & $6.7(7.6)$ & 0.22 & $0.3(-0.4$ to 1.1$)$ & 0.000 & 0.370 \\
\hline Aberrant Behavior Checklist - Irritability ${ }^{\mathrm{d}}$ & 84 & 41 & 43 & $9.3(13.5)$ & $7.1(7.5)$ & 0.14 & $0.2(-0.6$ to 1.0$)$ & 0.000 & 0.577 \\
\hline Modified Overt Aggression Scale ${ }^{d}$ & 83 & 41 & 42 & $7.0(15.9)$ & $6.5(13.8)$ & 0.01 & $-0.7(-12.2$ to 10.8$)$ & 0.000 & 0.903 \\
\hline $\begin{array}{l}\text { ndard deviation inflated for centre-level clusterir } \\
\text { ference calculated as: Control - Intervention. } \\
\text { acluster correlation coefficient (ICC): adjustment }\end{array}$ & 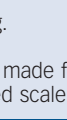 & 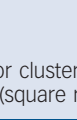 & t & nd & & 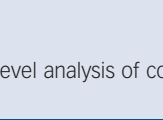 & ariance (ANCOVA) m & & \\
\hline
\end{tabular}

the $\mathrm{ABC}-\mathrm{H}$ and $\mathrm{ABC}-\mathrm{I}$ (Table 6). These effects increased slightly after excluding reports from keyworkers who also acted as lay therapists $(\mathrm{ABC}-\mathrm{H}, P=0.002$; ABC-I, $P=0.004)$, and after excluding non-adherent participants (CACE analysis: $P<0.001$ for both measures). Home carers also reported significant improvements in $\mathrm{ABC}-\mathrm{H}$ scores at 16-week follow-up.

\section{Mental health and related measures}

There were no significant differences in service users' self-reports on the measures of anxiety, depression, self-esteem or quality of life (Table 7).

\section{Costs}

The mean hourly cost of intervention per service user was $£ 25.26$. The mean hourly excess cost of intervention compared with treatment as usual was $\mathfrak{£} 12.34$, giving an overall excess cost of

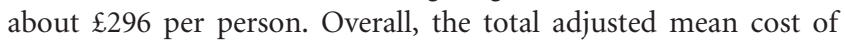
health and social care service use at 10-month follow-up, controlling for baseline levels, was lower for the intervention group by $£ 22.46$ per person per week, an amount almost equivalent to the weekly excess cost of the intervention. However, the difference was not statistically significant.

\section{Discussion}

\section{Main findings}

There was no significant effect of the intervention on the primary outcome measure self-reported anger as assessed by the Provocation Index. However, we did observe significant effects of the intervention on a range of secondary measures. Effect sizes were small to medium, but, as shown by the CACE analyses, these 


\begin{tabular}{|c|c|c|c|c|c|c|c|c|c|}
\hline \multirow[b]{2}{*}{ Outcome } & \multicolumn{3}{|c|}{$n$} & \multicolumn{2}{|c|}{ Mean (s.d.) $)^{a}$} & \multirow{2}{*}{$\begin{array}{l}\text { Standardised } \\
\text { effect size }\end{array}$} & \multirow{2}{*}{$\begin{array}{c}\text { Adjusted mean } \\
\text { difference }^{\mathrm{b}, \mathrm{c}}(95 \% \mathrm{Cl})\end{array}$} & \multirow[b]{2}{*}{$\mathrm{ICC}^{\mathrm{C}}$} & \multirow[b]{2}{*}{$P$} \\
\hline & Total & Contro & ervention & Control & Intervention & & & & \\
\hline \multicolumn{10}{|l|}{ Outcomes at 16 weeks } \\
\hline Glasgow Depression Scale ${ }^{d}$ & 157 & 81 & 76 & $9.8(6.8)$ & $9.1(8.2)$ & 0.19 & $0.2(-0.1$ to 0.5$)$ & 0.003 & 0.150 \\
\hline Glasgow Anxiety Scale & 154 & 79 & 75 & $18.3(8.7)$ & $16.0(10.7)$ & 0.17 & $1.6(-0.7$ to 3.9$)$ & 0.011 & 0.169 \\
\hline Rosenberg Self-Esteem Scale ${ }^{d}$ & 141 & 74 & 67 & $25.0(3.9)$ & $25.3(3.9)$ & 0.01 & $0.1(-0.2$ to 0.3$)$ & 0.000 & 0.514 \\
\hline Comprehensive Quality of Life ${ }^{d}$ & 129 & 67 & 62 & 99.9 (31.3) & $94.3(40.2)$ & 0.19 & $6.8(-4.8$ to 18.3$)$ & 0.000 & 0.249 \\
\hline \multicolumn{10}{|l|}{ Outcomes at 10 months } \\
\hline Glasgow Depression Scale ${ }^{d}$ & 144 & 72 & 72 & $8.1(6.0)$ & $8.3(8.2)$ & 0.07 & $0.1(-0.3$ to 0.5$)$ & 0.057 & 0.623 \\
\hline Glasgow Anxiety scale & 143 & 71 & 72 & $15.2(8.9)$ & $15.6(9.3)$ & 0.06 & $-0.5(-3.0$ to 1.9$)$ & 0.000 & 0.677 \\
\hline Rosenberg Self-Esteem Scale ${ }^{d}$ & 134 & 70 & 64 & $26.5(4.1)$ & $25.8(4.8)$ & 0.15 & $31.8(-39.2$ to 102.9$)$ & 0.069 & 0.362 \\
\hline Comprehensive Quality of Life ${ }^{d}$ & 140 & 70 & 70 & $98.1(41.9)$ & 97.5 (34.1) & 0.08 & $839.2(-2506.1$ to 4184.5$)$ & 0.000 & 0.621 \\
\hline
\end{tabular}

values were somewhat deflated by the inclusion of participants who attended few (or in some cases, no) sessions.

The significant effects included a self-report measure of anger in relation to situations that were identified as the most salient personal triggers for anger for each respondent. There are several important differences between the PACS-IPT and the Provocation Index, including the number of items ( $3 v .25)$, the rating of actual rather than hypothetical situations and the level of anger reported (see above). It was evident that personally salient events were captured poorly by the Provocation Index, and that many service users had difficulty in imagining some of the hypothetical situations envisaged in the Provocation Index. With hindsight, the Provocation Index was not an ideal primary outcome measure.

Keyworkers reported that the anger of service users in the intervention group had decreased significantly compared with the controls at both follow-up time points. Anger is conceptualised as an emotion with both internalising (emotional and cognitive) and externalising (behavioural) components. ${ }^{3,33}$ An analysis of our baseline data established that service users' self-ratings of anger are related to other self-ratings of mental health status, whereas carers' ratings of service-user anger are related to their ratings of observed challenging behaviour. ${ }^{34}$ This may explain why keyworkers reported larger effects, as service users showed clear differences on a number of behavioural outcomes.

The intervention decreased challenging behaviour, as rated by both keyworkers and home carers. It is well established that aggressive challenging behaviour can be minimised if staff implement appropriate behaviour-management methods, ${ }^{35,36}$ but to the best of our knowledge, this is the first controlled study to suggest improved self-management of aggressive challenging behaviour by people with intellectual disabilities. However, although significant effects were observed with the $\mathrm{ABC}$, a general measure of challenging behaviour, no significant improvement was reported using the MOAS, a more specific measure of aggression. Moreover, the effect was only observed immediately after the intervention and was not maintained at follow-up. This finding merits further study, including direct masked observational measurement of the number, intensity and nature of episodes of challenging behaviour.

Service users and keyworkers both reported significantly better utilisation of anger coping skills following the intervention. Keyworkers were not (and could not be) masked to group allocation, and this lack of independence in their ratings introduces a potential bias. However, lay therapists, who were perhaps the most likely to display bias, were no less stringent than other keyworkers in their ratings of the outcomes of the intervention, suggesting that bias in keyworker reports may not be a major issue. Earlier studies have reported increases in keyworker ratings of anger coping, ${ }^{16,17}$ but the service user version of the PACS was developed for the purpose of the present study, so self-ratings of anger coping have not previously been reported. The similar effect of the intervention on self- and carer ratings suggests that people with intellectual disabilities are able to report accurately on their own behaviour, and supports the validity of the service-user version of the PACS.

\section{Comparison with findings from other studies}

Although the intervention was successful in decreasing anger in personally relevant situations, decreasing keyworker ratings of service users' anger and increasing anger coping skills, we did not find a significant decrease in service users' self-ratings on the Provocation Index, unlike earlier studies that have used the Provocation Index or similar measures to evaluate comparable interventions delivered by clinical psychologists. ${ }^{14}$ The difference may simply reflect the greater methodological rigour of the present study. However, a number of other factors can be identified that could contribute to variability of outcome. Observation of group sessions revealed that most of the lay therapists were able to follow the manual and deliver the behavioural aspects of the programme, but struggled to work with service users on their emotions and cognitions. Also, lay therapists were instructed to emphasise to service users that, although anger should be expressed appropriately, anger is not wrong in itself and is often legitimate. Indeed, the stated aim of anger management interventions is to improve anger coping skills, not to decrease anger per se. Therefore, their success in teaching anger management skills that service users were able to use might be considered a better indication of a positive outcome than their more limited success in decreasing feelings of anger, which the intervention did not explicitly aim to do: indeed, the orientation of the manual may have mitigated against change on our primary outcome measure. It is possible that decreases in self-rated anger reported using the Provocation Index and similar instruments in psychologist-delivered interventions may reflect a more flexible, less manual-bound approach. However, it is important to emphasise that previous studies were potentially biased by researcher allegiance effects, ${ }^{37,38}$ and we can only speculate on the outcome of a large trial delivered by clinical psychologists less closely involved in the development of the intervention. 


\section{Costs}

Not surprisingly, the intervention cost a little more to deliver than treatment as usual. Although the mean adjusted cost difference was in favour of the intervention group, we cannot determine whether the excess cost of the intervention can be off-set by future savings in health and social care resource usage as the difference was not statistically significant. This may reflect an absence of power to detect such change due to the high variation in costs resulting from the inclusion of participants who lived in both residential placements (typically high cost) and family placements (typically low cost). Providing the indicated savings were not a statistical artefact, the excess costs of intervention would be recouped in a little over 3 months.

\section{Implications}

We believe that this is the first methodologically robust RCT of any CBT-based intervention for people with intellectual disabilities. The participants had not been referred to clinical psychology services but were readily identified by their day services as having the potential to benefit from the intervention. Although some doubts have been expressed about the feasibility of conducting RCTs with people with intellectual disabilities, ${ }^{39}$ we did not experience any significant difficulties in recruitment, retention or assessment, beyond what might be considered normal attrition of services and individuals.

Two major conclusions follow from this study. First, it demonstrates that people with intellectual disabilities can be taught by means of a brief and relatively inexpensive group-based intervention to cope with anger more appropriately. Second, it confirms that staff who work with people with intellectual disabilities can deliver a manualised intervention effectively with limited training. ${ }^{13,16}$ The lay therapists achieved positive outcomes and delivered the intervention with reasonable fidelity. The fidelity monitoring instrument we used was adapted from the Cognitive Therapy Scale for Psychosis. In a study of that instrument, only 8 of 14 psychotherapy trainees scored above $50 \%,{ }^{40}$ compared with our overall fidelity rating of $69 \%$ with $57 \%$ fidelity on the CBT components.

Care staff are an untapped resource that could be used to increase the availability of psychological interventions to people with intellectual disabilities, in line with the aim to increase their access to psychological therapies. ${ }^{11}$ The need for psychological interventions, as well as greater recognition of mental health problems among people with intellectual disabilities, is underlined by the high levels of untreated psychiatric caseness among the present participants. (Considering the size of the present sample and its geographical spread, we have no reason to think that the high levels of anxiety and depression reported are atypical of service users presenting with anger management problems.) However, given the high degree of variability between groups in both fidelity of delivery and outcomes, we recommend that staff-delivered interventions should wherever possible be closely supported, as here, by a qualified clinical psychologist.

\section{Funding}

This project was funded by the NIHR Health Technology Assessment (HTA) programme and will be published in full in the Health Technology Assessment journal series. Visit the HTA programme website for more details: www.hta.ac.uk/project/1925.asp. We also acknowledge the support provided for this study by the National Institute for Social Care and Health Research Clinical Research Centre (NISCHR CRC) and the colleagues from NISCHR and elsewhere not listed among the authors who assisted with data collection. The South East Wales Trials Unit is funded by NISCHR.
Paul Willner, DSC, Psychology Department, Swansea University, Swansea and Directorate of Learning Disability Services, Abertawe Bro Morgannwg University Health Board, Neath; John Rose, PhD, School of Psychology, University of Birmingham, Birmingham and Behavioural, Dementia and Psychological Services, Black Country Partnership NHS Foundation Trust, Stourbridge; Andrew Jahoda, PhD Institute of Health and Wellbeing, College of Medical, Veterinary and Life Sciences, University of Glasgow, Glasgow; Biza Stenfert Kroese, PhD, School of Psychology, University of Birmingham, Birmingham; David Felce, PhD, Welsh Centre for Learning Disabilities, Psychological Medicine and Neurology, Cardiff University, Cardiff; David Cohen, PhD, Health Economics and Policy Research Unit, University of Glamorgan, Pontypridd; Pamela MacMahon, DClinPsy, Institute of Health and Wellbeing, University of Glasgow, Glasgow; Aimee Stimpson, DClinPsy, Directorate of Learning Disability Services, Abertawe Bro Morgannwg University Health Board, Neath; Nicola Rose, DClinPsy, Behavioural, Dementia and Psychological Services, Black Country Partnership NHS Foundation Trust, Stourbridge; David Gillespie, BSC, South East Wales Trials Unit, Institute for Translation, Innovation, Methodology and Engagement (TIME), Cardiff University, Cardiff; Jennifer Shead, MSC, Behavioural, Dementia and Psychological Services, Black Country Partnership NHS Foundation Trust, Stourbridge; Claire Lammie, BSC, Institute of Health and Wellbeing, University of Glasgow, Glasgow; Christopher Woodgate, MSc, Directorate of Learning Disability Services, Glasgow; Christopher Woodgate, MSC, Directorate of Learning Disability Services,
Abertawe Bro Morgannwg University Health Board, Neath; Julia Townson, BA, Jacqueline Nuttall, BSc, Kerenza Hood, PhD, South East Wales Trials Unit, Institute for Translation, Innovation, Methodology and Engagement (TIME), Cardiff University, Cardiff, UK

Correspondence: P. Willner, Psychology Department, Swansea University, Swansea SA2 8PP, UK. Email: p.willner@swansea.ac.uk

First received 3 Dec 2012, final revision 19 Jan 2013, accepted 5 Feb 2013

\section{References}

1 Anderson CA, Bushman, BJ. Human aggression. Annu Rev Psychol 2002; 53: 27-51.

2 Novaco RW, Taylor JL. Assessment of anger and aggression in male offenders with developmental disabilities. Psychol Assess 2004; 16: 42-50.

3 Taylor JL, Novaco RW. Anger Treatment for People with Developmental Disabilities: A Theory, Evidence and Manual-Based Approach. Wiley, 2005.

4 Benson BA, Brooks WT. Aggressive challenging behaviour and intellectual disability. Curr Opin Psychiatry 2008; 21: 454-8.

5 Mills S, Rose J. The relationship between challenging behaviour, burnout and cognitive variables in staff working with people who have intellectual disabilities. J Intellect Disabil Res 2011; 55: 844-57.

6 Allen D, Hawkins S, Cooper V. Parents' use of physical interventions in the management of their children's severe challenging behaviour. J Appl Res Intellect Disabil 2006; 19: 356-63.

7 Hastings RP. Parental stress and behaviour problems of children with developmental disability. J Intellect Dev Disabil 2002; 27: 149-60.

8 Rose J, Jones F, Fletcher BC. Investigating the relationship between stress and worker behaviour. J Intellect Disabil Res 1998; 42: 163-72.

9 Hatton C, Wigham S, Craig J. Developing measures of job performance for support staff in housing services for people with intellectual disabilities. J Appl Res Intellect Disabil 2009; 22: 54-64.

10 Roth A, Fonagy P. What Works for Whom? A Critical Review of Psychotherapy Research. Guilford Press, 2004.

11 Department of Health/Care Service Improvement Partnership. Choices in Mental Health: Improving Access to Psychological Therapies. Department of Health, 2007.

12 McCabe MP, McGillivray JA, Newton DC. Effectiveness of treatment programmes for depression among adults with mild/moderate intellectual disability. J Intellect Disabil Res 2006; 50: 239-47.

13 McGillivray JA, McCabe MP, Kershaw MM. Depression in people with intellectual disability: an evaluation of a staff-administered treatment program. Res Dev Disabil 2008; 29: 524-36.

14 Willner $\mathrm{P}$, Jahoda A, Larkin P. Management of anger in people with intellectual disabilities. In Treatment for Anger in Specific Populations: Theory, Application, and Outcome (ed. E Fernandez). Oxford University Press, in press.

15 Rose J, Loftus M, Flint B, Carey L. Factors associated with the efficacy of a group intervention for anger in people with intellectual disabilities. Br J Clin Psychol 2005; 44: 305-17.

16 Willner $P$, Tomlinson $S$. Generalization of anger-coping skills from day-service to residential settings. J Appl Res Intellect Disabil 2007; 20: 553-62.

17 Willner $\mathrm{P}$, Brace $\mathrm{N}$, Phillips J. Assessment of anger coping skills in individuals with intellectual disabilities. J Intellect Disabil Res 2005; 49: 329-39.

18 Willner P, Jahoda A, Rose J, Stenfert-Kroese B, Hood K, Townson J, et al. Anger management for people with mild to moderate learning disabilities: 
study protocol for a multi-centre cluster randomised controlled trial of a manualised intervention delivered by day-service staff. Trials 2011; 12: 36.

19 Wechsler D. The Wechsler Abbreviated Scale of Intelligence. The Psychological Corporation, 1999.

20 Dunn LM, Sewell J, and Styles B. British Picture Vocabulary Scale (BPVS-III) (3rd edn). nferNelson, 2009.

21 Hatton C, Emerson E, Robertson J, Gregory N, Kessissoglou S, Perry J, et al. The adaptive behavior scale-residential and community (part I): towards the development of a short form. Res Dev Disabil 2001; 22: 273-88.

22 Mindham J, Espie CA. Glasgow Anxiety Scale for people with an Intellectual Disability (GAS-ID): development and psychometric properties of a new measure for use with people with mild intellectual disability. J Intellect Disabil Res 2003; 47: 22-30.

23 Cuthill FM, Espie CA, Cooper S-A. Development and psychometric properties of the Glasgow Depression Scale for people with a Learning Disability. Individual and carer supplement versions. Br J Psychiatry 2003; 182: 347-53.

24 Dagnan D, Sandhu S. Social comparison, self-esteem and depression in people with intellectual disability. J Intellect Disabil Res 1999; 43: 372-9.

25 Cummins RA. The Comprehensive Quality of Life Scale: Intellectual Disability (5th edn). Deakin University, 1997.

26 Aman MG, Singh NN, Stewart AW, Field CJ. The Aberrant Behavior Checklist: a behavior rating scale for the assessment of treatment effects. Am J Ment Defic 1985; 89: 485-91.

27 Oliver PC, Crawford MJ, Rao B, Reece B, Tyrer P. Modified Overt Aggression Scale (MOAS) for people with intellectual disability and aggressive challenging behaviour: a reliability study. J Appl Res Intellect Disabil 2007; 20: $368-72$.

28 Beecham J, Knapp MRJ. Costing psychiatric interventions. In Measuring Mental Health Needs (eds GJ Thornicroft, CR Brewin, JK Wing). Gaskell, 1992.

29 Quade D. Rank analysis of covariance. J Am Statist AssoC 1967; 62: 1187-200.
30 Borenstein M. Effect sizes for continuous data. In The Handbook of Research Synthesis and Metaanalysis (2nd edn) (eds H Cooper, LV Hedges, JC Valentine): 279-93. Russell Sage, 2009.

31 Jo B, Asparouhov T, Muthén BO. Intention-to-treat analysis in cluster randomized trials with noncompliance. Stat Med 2008; 27: 5565-77.

32 Robertson J, Emerson E, Pinkney L, Caesar E, Felce D, Meek A, et al. Quality and costs of community-based residential supports for people with mental retardation and challenging behavior. Am J Ment Retard 2004; 109: 332-44.

33 Novaco RW. Anger Control: The Development and Evaluation of an Experimental Treatment. Heath, 1975.

34 Rose J, Willner P, Shead J, Jahoda A, Gillespie D, Townson J, et al. Different factors influence self-reports and third-party reports of anger by adults with intellectual disabilities. J Appl Res Intellect Disabil, in press.

35 Rose N, Rose J, Kent S. Staff training in intellectual disability services: a review of the literature and implications for mental health services provided to individuals with intellectual disability. Int J Dev Disabil 2012; 58: 24-39.

36 Royal College of Psychiatrists, British Psychological Society and Royal College of Speech and Language Therapists. Challenging Behaviour: A Unified Approach. British Psychological Society, 2007.

37 Gaffan EA, Tsaousis I, Kemp-Wheeler SM. Researcher allegiance and meta-analysis: the case of cognitive therapy for depression. $J$ Consult Clin Psychol 1995; 63: 966-80.

38 Munder $\mathrm{T}$, Gerger $\mathrm{H}$, Trelle $\mathrm{S}$, Barth J. Testing the allegiance bias hypothesis: a meta-analysis. Psychother Res 2011; 21: 670-84.

39 Oliver PC, Piachaud J, Done J, Regan A, Cooray S, Tyrer P. Difficulties in conducting a randomized controlled trial of health service interventions in intellectual disability: implications for evidence-based practice. J Intellect Disabil Res 2002; 46: 340-5.

40 Haddock G, Devane S, Bradshaw T, McGovern J, Tarrier N, Kinderman P, et al. An investigation into the psychometric properties of the Cognitive Therapy Scale for Psychosis (CTSPsy). Behav Cogn Psychother 2001; 29: 221-33. 